\title{
Efficient red aggregation-induced emission based on bis(4-(N-(9- phenanthryl)-3,5-dimethylphenylamino)phenyl)fumaronitrile as organic nano-sized pigment
}

\author{
Wenguan Zhang ${ }^{1, a}$, Lian Qin ${ }^{1, b}$, Shengmin Zhao ${ }^{1, c}$ \\ ${ }^{1}$ Lab of Printing \& Packaging Material and Technology-Beijing Area Major Laboratory, \\ Beijing Institute of Graphic Communication, Beijing 102600, P. R. China

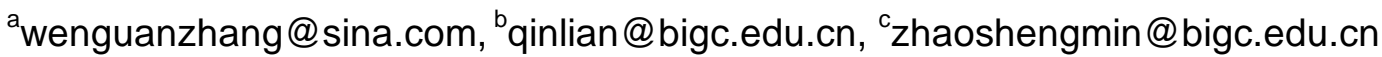

Keywords: Fumaronitrile; Phenanthrene; Aggregation-induced emission; Red Fluorescence; Solvatochromism.

\begin{abstract}
Many red fluorescent materials were weak and even no luminescence in solid state due to concentration quenching. So a novel red fluorescent pigment bis(4-(N-(9-phenanthryl)-3,5-dimethylphenylamino)phenyl)fumaronitrile (PhMPAFN) was synthesized. PhMPAFN with a pair of antiparallel dipole suppressing molecular interaction exhibited strong red photoluminescence at $650 \mathrm{~nm}$ in solid film. The decomposition temperature was found at $424{ }^{\circ} \mathrm{C}$. In different polarity of solvents the absorption and emission peaks of PhMPAFN showed positive and then negative solvatochromic shifts. PhMPAFN in tetrahydrofuran/water $(1: 1,1: 3,1: 5,1: 9$, v/v) was aggregated into fluorescent organic nanoparticles (FONs) in the range of 164 and $531 \mathrm{~nm}$ (coverage rate over $98 \%$ ). FONs displayed strong aggregation-induced emission at about $620 \mathrm{~nm}$ and had potential use in nano-sized fluorescence and optoelectronic device.
\end{abstract}

\section{Introduction}

Red-emissive light is very important in anti-counterfeit technology and full color organic light-emitting display (OLED). Red fluorescent molecules tend to be polar [1], or non-polar but extensively $\pi$-conjugated [2]. Many red emitters are highly emissive in solution, but prone to aggregation and crystallization inducing concentration quenching in solid state [3]. The fluorophores with arylamino diphenylfumaronitrile have a pair of antiparallel dipoles, for instance, bis(4-(N-(9,9-diethyl-2-fluorenyl)phenylamino)phenyl)fumaronitrile (EFPAFN) [4] and bis(4-(N-(9phenanthryl)-3,5-dimethylphenylamino)phenyl)fumaronitrile (PhMPAFN) [5,6], are rare red- emitting materials. EFPAFN in fluorescent organic nanoparticles (FONs) exhibit the strong aggregation-induced emission (AIE) [7] and are much stronger than in solution. FONs had potential use in nano-sized fluorescence and optoelectronic device.

In this work, red fluorescent pigment PhMPAFN was synthesized. In different polarity of solvents the positive and negative solvatochromism shift in absorption and emission was discussed. PhMPAFN in tetrahydrofuran/water was aggregated into fluorescent organic nanoparticles (FONs). The red AIE of FONs was investigated.

\section{Experimental}

Synthetic procedures. 9-(3,5-Dimethylphenylamino)phenanthrene, bis(4-bromophenyl)fumaronitrile, and bis(4-(N-(9-phenanthryl)-3,5-dimethylphenylamino)phenyl)fumaronitrile (PhMPAFN) were synthesized and organic light-emitting device (OLED) was characterized in the reference [6].

Instruments. UV-vis absorption, photoluminescence (PL) spectra and thermogravimetric analysis (TGA) were measured by Shimadzu UV-2501 PC, Perkin Elmer LS-55 luminescence spectrometer, and Netzsch TG-209, respectively. The diameter distribution of nanoparticles was performed by using Zeta Nano 90. 
Preparation of fluorescent organic nanoparticles (FONs). Red pigment PhMPAFN was dissolved by tetrahydrofuran (THF) to $10^{-5} \mathrm{~mol} / \mathrm{l}$ solution in a flask in an ultrasonic bath, a certain volume of deionized water was added dropwise into the above flask being vibrated for $15 \mathrm{~min}$. Fluorescent organic nanoparticles (FONs) were prepared.

\section{Results and discussions}

Characterization of synthesis and structure. Synthetic route and structure of PhMPAFN were showed in Fig. 1.

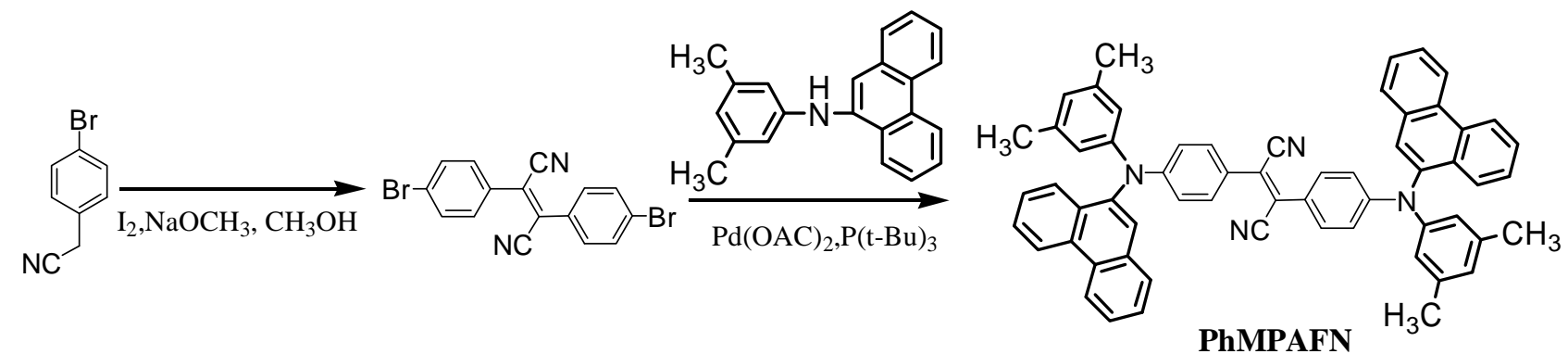

Fig. 1 Schematic diagram and structure of PhMPAFN

9-(3,5-Dimethylphenylamino)phenanthrene reacted with bis(4-bromophenyl)fumaronitrile by using palladium-catalyzed couplings such as $\mathrm{Pd}(\mathrm{OAc})_{2} / \mathrm{P}(\mathrm{t}-\mathrm{Bu})_{3}$ and a non-nucleophilic base $\mathrm{Cs}_{2} \mathrm{CO}_{3}$ to afford red fluorescent pigment bis(4-(N-(9-phenanthryl)-3,5-dimethylphenylamino)phenyl)fumaronitrile (PhMPAFN).

Thermal property. Thermal stability was tested by thermogravimetric analysis (TGA) at a heating rate of $10^{\circ} \mathrm{C} / \mathrm{min}$ from 25 to $650^{\circ} \mathrm{C}$ with a nitrogen flow. The thermal decomposition temperature (Td, onset) was located at $424{ }^{\circ} \mathrm{C}$ for PhMPAFN. It had the excellent thermal stability to be suitable for optoelectronic device.

Solvatochromic shift in absorption and emission spectra. UV-vis absorption and emission spectra of PhMPAFN in different polarity of solvents were discussed as shown in Fig. 2. In nonpolar carbon tetrachloride (TCC), increasing polarity from dichloromethane (DCM) to tetrahydrofuran (THF) to N, N-dimethylformamide (DMF), main absorption peaks of PhMPAFN were 480, 491, 481, and $483 \mathrm{~nm}$, respectively. This revealed positive, negative, and then positive solvatochromic shifts in absorption spectra.
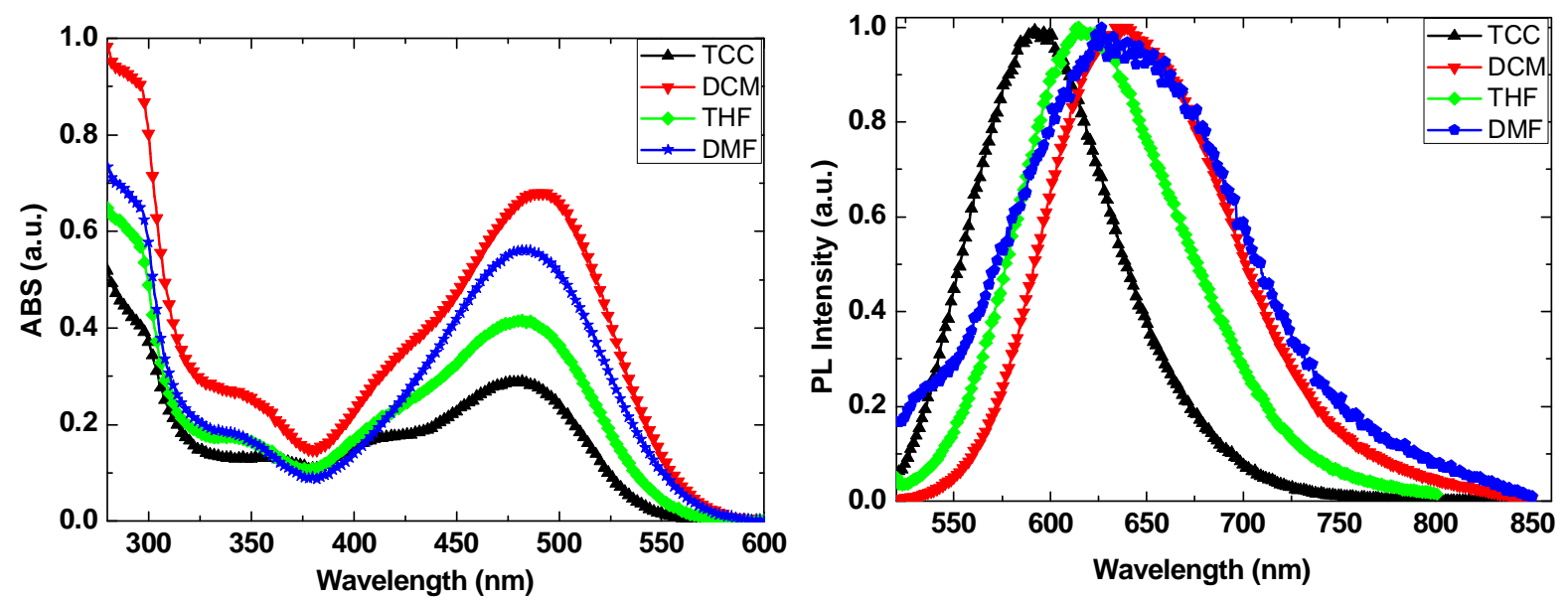

Fig. 2 UV-vis absorption (left) and emission spectra (right) of PhMPAFN in solvents 
In different polarity of TCC, DCM, THF and DMF, emission peaks of PhMPAFN were at 594, 638, 616, and $628 \mathrm{~nm}$, respectively. These showed positive, negative, and then positive solvatochromism in emission to indicate intramolecular electron transfer in PhMPAFN.

Dimensions distribution of fluorescent organic nanoparticles (FONs). PhMPAFN in THF/ water $(1: 1,1: 3,1: 5,1: 9$, v/v) mixture was aggregated into fluorescent organic nanoparticles (FONs) in the range of 164 and $531 \mathrm{~nm}$. The data of percentage distributions were listed in Table 1.

Table 1 Percentage distribution of particles

\begin{tabular}{|c|c|c|c|}
\hline \multirow{2}{*}{$\begin{array}{l}\text { THE/water } \\
\text { Size (nm) }\end{array}$} & $1: 3$ & $1: 5$ & $1: 9$ \\
\hline & \multicolumn{3}{|c|}{ Percentage distribution $(\%)$} \\
\hline 164.2 & 0.1 & 2.9 & 6.0 \\
\hline 190.1 & 1.4 & 7.3 & 10.6 \\
\hline 220.2 & 5.7 & 12.4 & 14.5 \\
\hline 255.0 & 11.9 & 16.6 & 17.1 \\
\hline 295.3 & 17.5 & 18.3 & 17.4 \\
\hline 342.0 & 20.1 & 16.9 & 14.8 \\
\hline 396.1 & 18.7 & 12.9 & 10.2 \\
\hline 458.7 & 14.0 & 7.7 & 5.2 \\
\hline 531.2 & 7.7 & 3.3 & 1.6 \\
\hline 615.1 & 2.5 & 0.9 & 0.2 \\
\hline
\end{tabular}

By analyzing the various volume ratios in FONs, when the proportion (v/v) of water was between 20 and $50 \%$, the percentage distribution of particle size ranged from 1 to $4 \mathrm{~nm}$. With the proportion of water from 75 to $90 \%$, the number and the diameter of aggregating particles gradually increased in the range of 164 and $531 \mathrm{~nm}$ (coverage rate over $98 \%$ ).

The emission of nanoparticles. The photoluminescent (PL) spectra of FONs for PhMPAFN in $\mathrm{THF} /$ water mixture were given in Fig. 3.

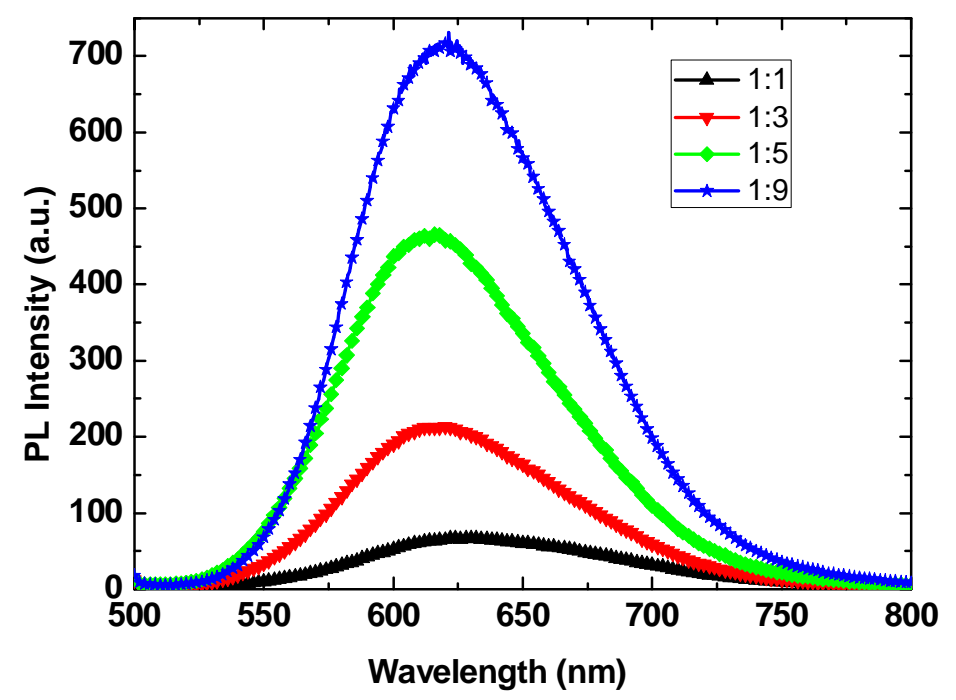

Fig. 3 The PL spectra of FONs of PhMPAFN

The emission intensity gradually increased and the main peak basically remained basically unchanged with the increasing of particle size. The PL spectra of FONs for PhMPAFN appeared at $620 \mathrm{~nm}$. As a whole, FONs for PhMPAFN showed highly efficient red AIE and had potential application in nano-sized fluorescence and optoelectronic device. 


\section{Conclusions}

A red fluorescent pigment PhMPAFN was prepared, which restrained molecular interaction to show strong red photoluminescence at $650 \mathrm{~nm}$ in solid film. The Td was found at $424{ }^{\circ} \mathrm{C}$. PhMPAFN in various solvents displayed solvatochromic shifts in the absorption and emission. PhMPAFN in THF/water (1:3, 1:5, 1:9, v/v) was aggregated into FONs between 164 and $531 \mathrm{~nm}$. FONs exhibited strong red AIE at $620 \mathrm{~nm}$ and had promising use in nano-sized emission and optoelectronic device.

\section{Acknowledgments}

This work was funded by the Beijing Commission of Education Grants (KM201310015004).

\section{References}

[1] X.T. Tao, S. Miyata, H. Sasabe, G.J. Zhang, Efficient organic red electroluminescent device with narrow emission peak, Appl. Phys. Lett. 78 (2001) 279-281.

[2] M.A. Baldo, D.F. O'Brien, Y.You, A. Shoustikov, S. Sibley, M.E. Thompson, S.R. Forrest, Highly efficient phosphorescent emission from organic electroluminescent devices, Nature 395 (1998) 151- 154.

[3] B.J. Chen, X.W. Sun, X.Q. Lin, C.S. Lee, S.T. Lee, Improved luminescent efficiency of a red organic dye with modified molecular structure, Materials Science and Engineering: B 100(1) (2003) 59-62.

[4] W.G. Zhang, Z.Q. He, L.P. Mu, Y. Zou, Y.S. Wang, S.M. Zhao, Red non-doped electroluminescent dyes based on arylamino fumaronitrile derivatives, Dyes pigments 85 (2010) 86-92.

[5] H.C. Yeh, S.J. Yeh, C.T. Chen, Readily synthesised arylamino fumaronitrile for non-doped red organic light-emitting diodes, Chem. Commun. (2003) 2632-2633.

[6] W.G. Zhang, Z.Q. He, Y.S. Wang, S.M. Zhao, Non-doped red-green-blue electroluminescent devices based on fluorenyl and phenanthryl phenylamino derivatives, Thin Solid Films 562 (2014) 299-306.

[7] W.G. Zhang, L. Qin, S.M. Zhao, Organic nano-sized pigment based on bis(4-(N-(9,9-diethyl2-fluorenyl)phenylamino)phenyl)fumaronitrile as highly efficient red fluorescence, Advanced Materials Research 197-198 (2011) 290-293. 